\title{
Evaluation of Image Registration in PET/CT of the Liver and Recommendations for Optimized Imaging
}

Wouter V. Vogel ${ }^{1}$, Jorn A. van Dalen ${ }^{1}$, Bas Wiering ${ }^{2}$, Henkjan Huisman ${ }^{3}$, Frans H.M. Corstens ${ }^{1}$, Theo J.M. Ruers ${ }^{2}$, and Wim J.G. Oyen ${ }^{1}$

${ }^{1}$ Department of Nuclear Medicine, Radboud University Nijmegen Medical Center, Nijmegen, The Netherlands; ${ }^{2}$ Department of Surgery, Radboud University Nijmegen Medical Center, Nijmegen, The Netherlands; and ${ }^{3}$ Department of Radiology, Radboud University Nijmegen Medical Center, Nijmegen, The Netherlands

Multimodality PET/CT of the liver can be performed with an integrated (hybrid) PET/CT scanner or with software fusion of dedicated PET and CT. Accurate anatomic correlation and good image quality of both modalities are important prerequisites, regardless of the applied method. Registration accuracy is influenced by breathing motion differences on PET and CT, which may also have impact on (attenuation correction-related) artifacts, especially in the upper abdomen. The impact of these issues was evaluated for both hybrid PET/CT and software fusion, focused on imaging of the liver. Methods: Thirty patients underwent hybrid PET/CT, 20 with CT during expiration breathhold (EB) and 10 with CT during free breathing (FB). Ten additional patients underwent software fusion of dedicated PET and dedicated expiration breath-hold CT (SF). The image registration accuracy was evaluated at the location of liver borders on CT and uncorrected PET images and at the location of liver lesions. Attenuation-correction artifacts were evaluated by comparison of liver borders on uncorrected and attenuationcorrected PET images. CT images were evaluated for the presence of breathing artifacts. Results: In EB, $40 \%$ of patients had an absolute registration error of the diaphragm in the craniocaudal direction of $>1 \mathrm{~cm}$ (range, -16 to $44 \mathrm{~mm}$ ), and $45 \%$ of lesions were mispositioned $>1 \mathrm{~cm}$. In $50 \%$ of cases, attenuationcorrection artifacts caused a deformation of the liver dome on PET of $>1 \mathrm{~cm}$. Poor compliance to breath-hold instructions caused CT artifacts in $55 \%$ of cases. In FB, $30 \%$ had registration errors of $>1 \mathrm{~cm}$ (range, -4 to $16 \mathrm{~mm}$ ) and PET artifacts were less extensive, but all CT images had breathing artifacts. As SF allows independent alignment of PET and CT, no registration errors or artifacts of $>1 \mathrm{~cm}$ of the diaphragm occurred. Conclusion: Hybrid PET/CT of the liver may have significant registration errors and artifacts related to breathing motion. The extent of these issues depends on the selected breathing protocol and the speed of the CT scanner. No protocol or scanner can guarantee perfect image fusion. On the basis of these findings, recommendations were formulated with regard to scanner requirements, breathing protocols, and reporting.

Received Sep. 21, 2006; revision accepted Mar. 9, 2007.

For correspondence or reprints contact: Wouter V. Vogel, MD, Department of Nuclear Medicine (565), Radboud University Nijmegen Medical Center, Postbox 9101, 6500 HB Nijmegen, The Netherlands.

E-mail: w.vogel@nucmed.umcn.nl

COPYRIGHT @ 2007 by the Society of Nuclear Medicine, Inc.
Key Words: PET; PET/CT; accuracy; liver imaging; oncology

J Nucl Med 2007; 48:910-919

DOI: 10.2967/jnumed.107.041517

A ccurate imaging of liver metastases is important for clinical decision making when considering locoregional therapy, such as partial liver resection or radiofrequency ablation $(1,2)$. These interventions rely on accurate information about the localization and the extent of tumor sites $(3,4)$. The added value of functional imaging with ${ }^{18} \mathrm{~F}-\mathrm{FDG}$ PET to conventional anatomic imaging (CT, especially, and MRI) has been well recognized, especially when assessing previous therapeutic interventions $(5,6)$. However, the exact localization of lesions on ${ }^{18} \mathrm{~F}$-FDG PET is limited by a relatively low spatial resolution and a lack of anatomic reference. The obvious benefit of combining the capabilities of CT (anatomic reference) and ${ }^{18}$ F-FDG PET (sensitive tumor detection) has led to the practice of correlation of images as obtained by PET and by CT (7-9). Correlation can be performed with mere visual side-by-side evaluation of images acquired by separate scanners or with integrated images provided by either an integrated (hybrid) PET/CT scanner or software image fusion of dedicated PET and CT (10). Regardless of the methodology, the anatomic correlation of both image sets must be accurate. This implies that the liver needs to be in the same anatomic position and shape during both CT and PET acquisitions. However, CT and PET are influenced differently by breathing motion. As free breathing is mandatory for PET acquisition, PET has blurring in the lower thoracic and upper abdominal areas. $\mathrm{CT}$ acquisition must be adapted to match these images, by scanning during free breathing or timed unforced expiration (10), but neither approach fully eliminates the risk of registration errors between PET and CT $(11,12)$. Furthermore, these registration errors can introduce artifacts on PET images in hybrid PET/CT, where attenuation correction of PET images is based on the CT images. Such artifacts may 
compromise both clinical interpretation and quantitative evaluation of PET images (13).

Diagnostic imaging requires optimal image quality. In this study, we determined the extent of anatomic registration errors and the occurrence of artifacts in hybrid PET/CT of the liver using a robust technique, during different breathing protocols, and performed a direct comparison with software image fusion of separately acquired PET and CT. According to our findings, recommendations were formulated with regard to scanner requirements, breathing protocols, and reporting.

\section{MATERIALS AND METHODS}

Integrated PET/CT images were acquired with 3 different protocols. Twenty consecutive patients with suspected metastases from colorectal cancer underwent hybrid PET/CT with low-dose CT during expiration breath-hold (EB). Ten other consecutive patients (March 2006), who were referred for various indications and who were unable to comply with breathing instructions for various reasons, underwent hybrid PET/CT with low-dose CT during free breathing (FB). Ten more consecutive patients (between December 2002 and November 2003) with suspected metastases from colorectal carcinoma underwent software fusion of dedicated PET and dedicated diagnostic CT acquired during breath-hold (SF).

\section{Image Acquisition}

Hybrid PET/CT scans were acquired using a Biograph Duo (Siemens Medical Solutions USA, Inc.) containing a 2-slice CT scanner. A low-dose CT scan for localization and attenuationcorrection purposes was acquired in the caudocranial direction from the thighs to the base of the skull. Scanning parameters included $40 \mathrm{~mA} \cdot \mathrm{s}, 130 \mathrm{kV}$, 5-mm slice collimation, 0.8 -s rotation time, and pitch of 1.5 , reconstructed to 3-mm slices for smooth coronal representation. CT scans were acquired during timed unforced expiration breath-hold (EB) or during free breathing (FB). Timed expiration breath-hold consisted of free breathing during the first (caudal) part of the scan, a deep inspiration command at the level of the spina iliaca superior, immediately followed by a command to expire and breath-hold; patients were allowed to resume free breathing at the level of the lung tops. The total expiration breath-hold time was about $30 \mathrm{~s}$. Free breathing was performed without specific patient instructions. No intravenous contrast was applied. For PET, a 3-dimensional (3D) emission scan of the central body was acquired during free breathing, $60 \mathrm{~min}$ after intravenous injection of $250 \mathrm{MBq}{ }^{18} \mathrm{~F}$-FDG. The acquisition time per bed position was $4 \mathrm{~min}$ for emission only. Uncorrected emission images as well as images with CT-based attenuation correction were reconstructed, both using 2 iterations, 8 subsets, and a $5-\mathrm{mm}$ 3D gaussian filter. Dedicated ${ }^{18}$ F-FDG PET scans were acquired using an ECAT Exact 47 scanner (Siemens Medical Solutions). A 3 D emission scan was acquired and reconstructed identical to PET from PET/CT. In addition, a 2-dimensional ${ }^{68} \mathrm{Ge}$-based transmission scan was acquired for attenuation correction. The acquisition time per bed position was $5 \mathrm{~min}$ for emission and $3 \mathrm{~min}$ for the transmission. Dedicated CT scans were acquired using a Somatom Volume Zoom (Siemens Medical Solutions) 4-slice scanner. Scans of the liver were acquired with $80 \mathrm{mAs}, 130 \mathrm{kV}, 0.5$-s rotation time, and 5-mm slice thickness, during unforced expiration breath-hold.
Intravenous contrast was applied; the portal-phase images were selected for image fusion with PET.

\section{Image Registration Procedure}

For hybrid PET/CT, normal image registration quality-assurance procedures were followed as described by the manufacturer. This involved alignment of the PET and CT gantries after maintenance, using a "crossed-lines" phantom. No additional image registration optimization was performed after scanning. Software image registration was performed on a personal computer with image viewing and registration software, developed in-house, based on the visualization toolkit VTK (14) and the insight segmentation and registration toolkit ITK (15). The procedure has been described in more detail previously (16). In brief, the software allows rigidbody image registration based on 3 translation and 3 rotation parameters. Anatomic registration of PET emission images to CT was pursued using an implementation of the automatic mutual information algorithm, on a $3 \mathrm{D}$ volume of interest containing the liver.

\section{Image Analysis}

Image sets from PET and CT were correlated through evaluation of borders of the liver and focal lesions within the liver. Mismatches of $>10 \mathrm{~mm}$ were considered potentially clinically relevant. Mismatches of focal lesions were expressed as 3D vectors. For liver borders, this approach is not possible, because a unidirectional shift of a liver border may be complicated by a (unrecognizable) deformation or rotation that alters the location that represents the top. Selected for landmarks were the tangent points (tops) of 3 liver borders: the diaphragmatic dome, the right liver border, and the caudal tip. 3D ellipsoids were manually positioned to match the curved shapes of the liver borders (Fig. 1); the locations of the tangent points were then derived mathematically. Mismatches were expressed as 1-dimensional distances along the axis of the largest movement (e.g., the craniocaudal direction for the diaphragmatic dome and the caudal tip of the liver; the lateral direction for the right lateral liver border). This procedure was performed separately on CT, uncorrected (UPET), and attenuationcorrected (acPET) images, blinded from each other.

The localization of liver borders is difficult on UPET and acPET, as the images are blurry. The selected visual cutoff for positioning of a border may be different for uPET and acPET images. The observer-specific systematic bias between localization of liver borders on UPET and acPET was determined by comparing images from dedicated PET, where the position of the liver is theoretically identical on both image sets. The true position of the liver border was assumed to be between the visual localizations on uPET and acPET. All uPET and acPET measurements were corrected afterward for this bias, using the average measurement difference from the theoretic position. The interobserver variability for manual determination of positional differences of tangent points, after correction of the systematic bias, was evaluated in 5 subsequent dedicated PET scans (both uPET and acPET) by 2 experienced observers.

Definitions. CT images, uPET images, and acPET images were evaluated for image registration errors, attenuation-correction artifacts, and the visual discernibility of these errors.

- Registration errors: The relative anatomic/positional mismatch of structures (either circumscript lesions or organ borders) as visible on uPET and CT images, expressed as a distance in millimeters. 
FIGURE 1. Localization of liver borders. Coronal slices of CT (A), attenuationcorrected PET $(B)$, and uncorrected PET (C) of a single patient, acquired with hybrid PET/CT during expiration breath-hold. Circles represent slices through 3D ellipsoids that were mapped to diaphragmatic dome (green), right lateral border (blue), and caudal tip (red) to determine differences in their respective positions.
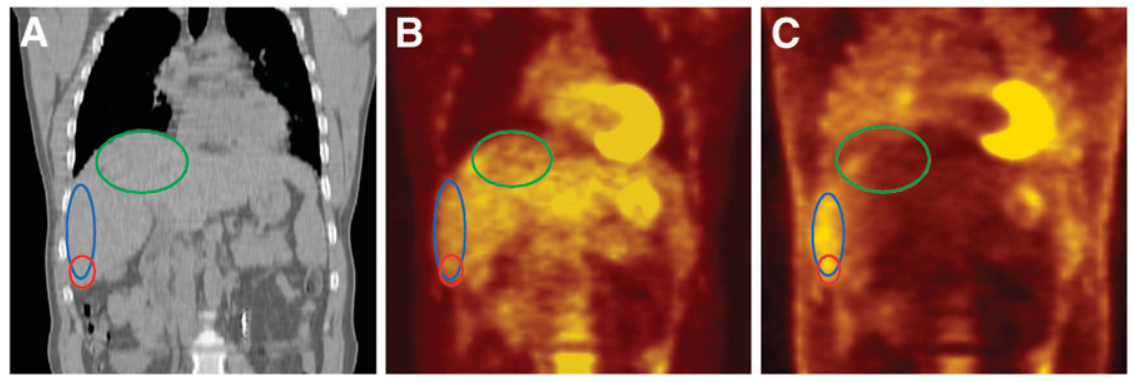

- Attenuation-correction artifacts: Contour changes of structures as visible on PET images before and after attenuation correction-that is, a difference of liver border positions between uPET and acPET images, expressed as a distance in millimeters.

- Visible errors: Mismatch of visible structures (either circumscript lesions or organ borders) between acPET and CT images, representing the extent to which the combination of registration errors and attenuation-correction errors can be recognized and appreciated on acPET images, expressed as a distance in millimeters.

Analyzed Parameters.

- Registration errors of liver borders: The error in local image registration was determined for the 3 liver borders separately, for all EB, FB, and SF images. Differences in image registration between imaging techniques were evaluated using the Bartlett test for equality of variances (level of significance, $0.05)$.

- Registration errors of liver lesions: Focal lesions were evaluated using CT and acPET images, for EB and SF. Lesions were considered evaluable when the center could be identified on both CT and acPET. This analysis was not possible on free-breathing CT images, as breathing motion artifacts on CT prevented reliable determination of the center of lesions. The interobserver variation in manual localization of lesion centers on $\mathrm{CT}$ and acPET was evaluated for 5 subsequent lesions on CT and acPET by 2 experienced observers.

- Attenuation-correction artifacts: The extent of attenuationcorrection artifacts on PET was evaluated for all liver borders, for EB, FB, and SF. The apparent position of the liver borders (tangent points) was determined before and after attenuation correction (i.e., on UPET and acPET images, respectively), similar to the evaluation of registration errors. Differences in the extent of attenuation-correction artifacts between protocols were evaluated using the Bartlett test for equality of variances (level of significance, 0.05).

- Visible errors on acPET: The extent to which the combination of localization errors and attenuation-correction errors, as detected previously, were discernible on acPET was evaluated for all liver borders, by comparing acPET and CT in an approach similar to that used for the assessment of registration errors. This analysis was performed for EB, FB, and SF.

- Breathing artifacts on CT: Artifacts caused by breathing motion may be depicted on CT images as locoregional deformities of the liver (i.e., breath-hold not sustained) or as deformities throughout the liver (i.e., free breathing). The presence of both types of artifacts was evaluated visually for all CT images. Qualitative analysis of these artifacts was not attempted.

\section{RESULTS}

All FB scans, SF scans, and all registration procedures were performed without problems. Of the $20 \mathrm{~EB}$ patients, 11 patients did not fully comply with an adequate breathhold during CT acquisition of the whole liver range. This was visible on CT images as various artifacts; a more detailed evaluation is provided below. Therefore, the original EB group $\left(\mathrm{EB}_{\text {all }}\right)$ was divided into 9 patients with adequate breath-hold $\left(\mathrm{EB}_{\text {adequate }}\right)$ and 11 patients with inadequate breath-hold $\left(\mathrm{EB}_{\text {failed }}\right)$ for additional separate analysis.

\section{Registration Errors of Liver Borders}

The average absolute interobserver variability in determination of liver border position differences on uPET and acPET, measured in 1 direction, was $2 \mathrm{~mm}$ (range, -3 to 4 $\mathrm{mm}$; SD, $3 \mathrm{~mm}$ ) for the diaphragmatic dome, $2 \mathrm{~mm}$ (range, -3 to $2 \mathrm{~mm}$; SD, $2 \mathrm{~mm}$ ) for the right lateral border, and $2 \mathrm{~mm}$ (range, -4 to $3 \mathrm{~mm}$; SD, $3 \mathrm{~mm}$ ) for the caudal tip.

For $\mathrm{EB}_{\text {all }}$, the average absolute image registration error at the diaphragmatic dome of the liver in the craniocaudal direction was $11 \mathrm{~mm}$ (relative range, -16 to $+44 \mathrm{~mm}$ in $40 \%$ of cases $>10 \mathrm{~mm}$ ). Visual inspection revealed that the largest errors were caused by expiration during breath-hold CT that was not deep enough. For the caudal tip of the liver, the average error was $19 \mathrm{~mm}$ (range, $0-53 \mathrm{~mm}$ in $55 \%$ of cases $>10 \mathrm{~mm}$ ). Registration errors were all $<10 \mathrm{~mm}$ at the right lateral liver border and were $<10 \mathrm{~mm}$ at all liver borders in the FB and SF protocols. The image registration errors of FB and SF at the locations of the diaphragmatic dome and caudal tip were significantly less than those of $\mathrm{EB}_{\text {all }}(P<0.05)$. SF did not perform significantly better than FB at the location of all liver borders. The results are listed in more detail in Table 1. The distribution of registration errors peracquisition protocol is represented in Figure 2.

The image registration at the diaphragmatic dome in breath-hold PET/CT was not significantly influenced by the adequacy of the breath-hold instructions during CT (error $>$ $10 \mathrm{~mm}$ in $44 \%$ of $\mathrm{EB}_{\text {adequate }}$ and in $36 \%$ of $\mathrm{EB}_{\text {failed }}$; not significant). Conversely, the registration of the caudal tip of the liver appeared to be influenced by the success of the 
TABLE 1

Extent of Registration Errors and Attenuation-Correction Artifacts

\begin{tabular}{|c|c|c|c|c|c|c|}
\hline \multirow[b]{2}{*}{$\begin{array}{l}\text { Breathing } \\
\text { protocol }\end{array}$} & \multirow[b]{2}{*}{$\begin{array}{l}\text { Measured } \\
\text { landmark }\end{array}$} & \multirow[b]{2}{*}{$\begin{array}{l}\text { Measurement } \\
\text { direction }\end{array}$} & \multicolumn{2}{|c|}{ Registration errors } & \multicolumn{2}{|c|}{ Attenuation-correction artifacts } \\
\hline & & & $\begin{array}{l}\text { Range } \\
(\mathrm{mm})\end{array}$ & $\begin{array}{c}\text { Absolute } \\
\text { mean }(\mathrm{mm})\end{array}$ & $\begin{array}{l}\text { Range } \\
(\mathrm{mm})\end{array}$ & $\begin{array}{l}\text { Absolute mean } \\
(\mathrm{mm})\end{array}$ \\
\hline \multirow[t]{4}{*}{ EB } & Diaphragmatic dome & $z$-axis & -16 to 44 & 11 & -18 to 41 & 11 \\
\hline & $\mathrm{R}$ lateral border & $x$-axis & -8 to 8 & 5 & -4 to 10 & 2 \\
\hline & Caudal tip & $z$-axis & -3 to 53 & 19 & -6 to 4 & 2 \\
\hline & Individual liver lesions & $3 \mathrm{D}$ vector & 3 to 24 & 11 & & NA \\
\hline \multirow[t]{4}{*}{ FB } & Diaphragmatic dome & $z$-axis & -4 to 16 & 7 & -7 to 11 & 6 \\
\hline & $\mathrm{R}$ lateral border & $x$-axis & -4 to 7 & 3 & -4 to 4 & 2 \\
\hline & Caudal tip & $z$-axis & -5 to 20 & 9 & -4 to 4 & 2 \\
\hline & Individual liver lesions & $3 \mathrm{D}$ vector & & NA & & NA \\
\hline \multirow[t]{4}{*}{ SF } & Diaphragmatic dome & $z$-axis & -3 to 8 & 3 & -3 to 5 & 3 \\
\hline & $\mathrm{R}$ lateral border & $x$-axis & -1 to 9 & 3 & -2 to 2 & 1 \\
\hline & Caudal tip & $z$-axis & -3 to 12 & 5 & -3 to 4 & 2 \\
\hline & Individual liver lesions & $3 \mathrm{D}$ vector & 7 to 14 & 9 & & NA \\
\hline$A=$ & ole. & & & & & \\
\hline
\end{tabular}

breath-hold procedure: error $>10 \mathrm{~mm}$ in $33 \%$ of $\mathrm{EB}_{\text {adequate }}$ and in $73 \%$ of $\mathrm{EB}_{\text {failed. However, due to the sample size, }}$ this difference did not reach statistical significance. When $\mathrm{EB}_{\text {all }}$ was limited to $\mathrm{EB}_{\text {adequate, }}$ there was no difference with FB in the diaphragmatic dome. This illustrates that using a faster CT scanner (with more likely successful completion of the breath-hold instructions) does not improve image registration in general, except in the region of the caudal tip where instructions tend to be executed when using a slow CT scanner.

\section{Registration Errors of Liver Lesions}

The average interobserver variability in localization of focal liver lesions, measured as a 3D vector, was $2 \mathrm{~mm}$ (range, 1-3 $\mathrm{mm}$ ) on CT and $1 \mathrm{~mm}$ (range, 0-2 mm) on acPET.

For $\mathrm{EB}_{\mathrm{all}}$, the average displacement of 11 detected lesions was $11 \mathrm{~mm}$ (range, 3-24 mm), with 5 lesions (45\%)

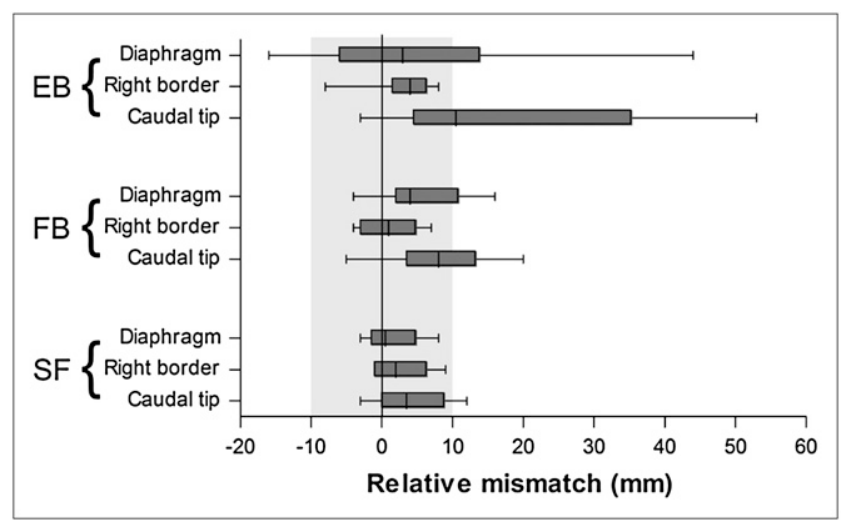

FIGURE 2. Image registration errors of liver borders. Relative image registration errors at location of several liver borders for EB (hybrid PET/CT with breath-hold CT), FB (hybrid PET/CT with free-breathing CT), and SF (software fusion of dedicated PET and CT). Registration errors occur primarily in craniocaudal direction (diaphragm and caudal tip affected most) because of insufficient expiration during CT. being displaced $>10 \mathrm{~mm}$. There were insufficient evaluable lesions for separate analysis of $\mathrm{EB}_{\text {adequate }}$ and $\mathrm{EB}_{\text {failed. For }}$ $\mathrm{SF}$, the average displacement of 5 detected lesions was 9 $\mathrm{mm}$ (range, 7-14 mm), with 1 lesion displaced $>10 \mathrm{~mm}$. Because of the limited number of evaluable lesions, statistical comparison of EB and SF was not performed. An example of a displaced lesion on hybrid PET/CT during EB is shown in Figure 3.

\section{Attenuation-Correction Artifacts}

For $\mathrm{EB}_{\text {all }}$, the average size of attenuation artifacts at the diaphragmatic liver dome in the craniocaudal direction was $11 \mathrm{~mm}$ (range, $0-41 \mathrm{~mm}$; in $50 \%$ of cases $>10 \mathrm{~mm}$ ) and was congruent with local registration errors (Fig. 4). Visual inspection again revealed that the largest artifacts were due to expiration that was not deep enough during breathhold CT. The occurrence of clinically relevant attenuationcorrection artifacts at the diaphragmatic dome did not depend on whether the patient successfully completed the CT breath-hold instructions (error $>10 \mathrm{~mm}$ in $45 \%$ of $\mathrm{EB}_{\text {adequate }}$ and in $56 \%$ of $\mathrm{EB}_{\text {failed }}$; not significant). This illustrates that the artifacts are unavoidable, even when using a fast CT scanner. An example of liver deformation due to attenuation correction in breath-hold hybrid PET/CT is shown in Figure 5.

For FB, the average attenuation artifact at the diaphragmatic liver dome measured $6 \mathrm{~mm}$ (range, 0-11 mm; 20\% $>10 \mathrm{~mm}$ ), both in the cranial and caudal direction. For SF, attenuation-correction artifacts of the liver are theoretically not an issue. Control measurements at the diaphragmatic dome showed an average absolute error of $3 \mathrm{~mm}$ (range, 0 $8 \mathrm{~mm}$; thus, in all patients within $10 \mathrm{~mm}$ ).

For all EB, FB, and SF cases, no significant attenuationcorrection artifacts occurred at the lateral border or the caudal tip. 

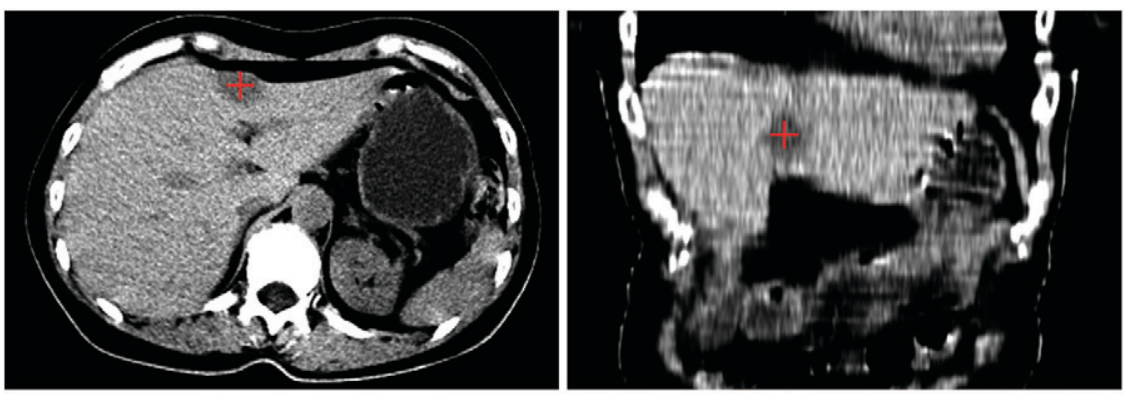

FIGURE 3. Misregistration of a liver lesion on breath-hold PET/CT. Transverse (left) and coronal (right) images of large liver metastasis in hybrid PET/CT with breath-hold CT. Center of lesion is marked with red cross on CT and with blue cross on PET. Positioning differences of liver between PET and CT acquisition resulted in mismatch of $13 \mathrm{~mm}$, measured as a 3D vector.
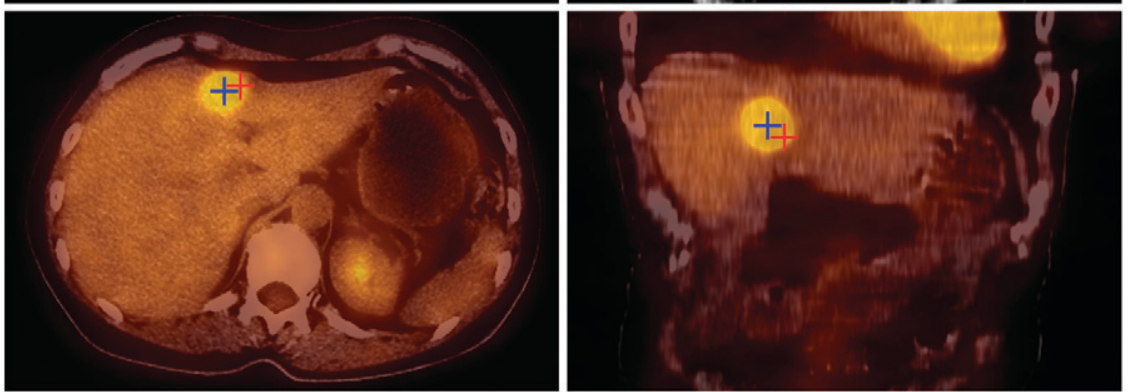

The extent of attenuation-correction artifacts at the diaphragmatic dome was significantly worse in $\mathrm{EB}_{\text {all }}$ than in $\mathrm{FB}$ or $\mathrm{SF}(P<0.05)$. When $\mathrm{EB}_{\text {all }}$ was limited to $\mathrm{EB}_{\text {adequate, }}$ the difference with $\mathrm{FB}$ in the diaphragmatic dome was not significant. FB was significantly worse than SF $(P<0.05)$. No clinically relevant attenuation-correction errors occurred at the right lateral border and the caudal tip with either technique.

\section{Visible Errors on acPET}

At the locations of the diaphragm and the right lateral liver border, no cases showed a visually discernible mismatch of $>10 \mathrm{~mm}$ at the liver border between acPET and $\mathrm{CT}$-for all EB, FB, and SF images-regardless of the

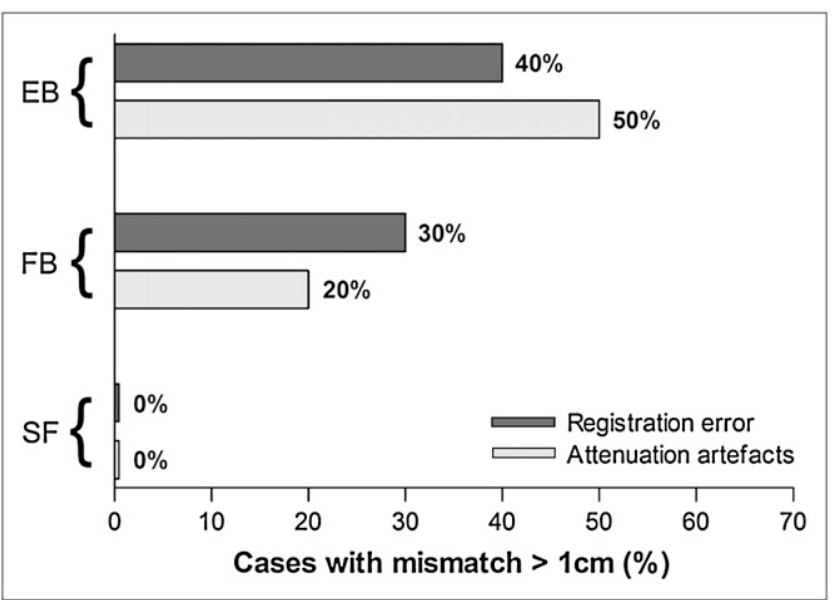

FIGURE 4. Errors at diaphragmatic dome. Extent of image registration errors and attenuation-correction artifacts of $>10$ $\mathrm{mm}$ was comparable at location of diaphragmatic dome of liver for EB (hybrid PET/CT with breath-hold CT), FB (hybrid PET/CT with free-breathing $\mathrm{CT}$ ), and SF (software fusion of dedicated PET and CT). presence of registration or attenuation-correction artifacts of $>10 \mathrm{~mm}$. Visually discernible errors were seen at the location of the caudal tip in all image series, with values similar to the local image registration error.

\section{Breathing Artifacts on CT}

In $\mathrm{EB}_{\text {all }}$, locoregional breathing artifacts in the liver were detected on the CT images of 4 patients (20\%), all attributable to breathing motion during acquisition despite instructions to hold the breath. Furthermore, the caudal tip of the right liver lobe appeared displaced or deformed in 7 additional patients $(35 \%)$, all attributable to the breathing instructions given when approaching the region of the liver in the caudocranial scanning direction. In FB, free-breathing artifacts were discernible throughout the images for all patients. No breathing artifacts were detected in the CT images used for SF. Examples of breathing artifacts on CT are shown in Figure 6 . The clinically relevant $(>10 \mathrm{~mm})$ breathing artifacts on CT are summarized in Table 2.

\section{DISCUSSION}

The results of these investigations illustrate that in the current implementation of integrated PET/CT, there is always a significant risk of clinically relevant registration errors and attenuation-correction errors when imaging the liver. The extent of these errors cannot be seen on PET images that have been corrected for photon attenuation using CT; this has implications for reviewing.

Some remarks must be made with regard to the accuracy of the performed measurements. Evaluation of PET/CT image registration and artifacts of the liver is not trivial, because the organ lacks well-defined, clearly discernible landmarks on PET. Evaluations are limited to liver borders and, if present, focal lesions within the liver. Evaluation of liver borders is restricted to those areas with sufficient contrast to 

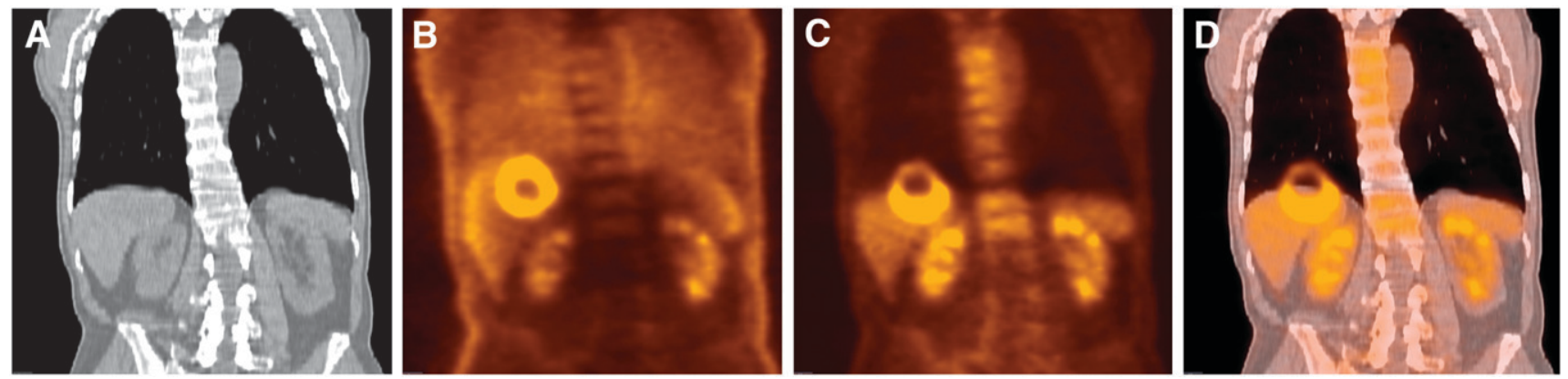

FIGURE 5. PET/CT attenuation-correction artifacts. Hybrid PET/CT of large liver metastasis with central necrosis, with CT acquired during expiration breath-hold: coronal slices of CT (A), uncorrected PET (B), attenuation-corrected PET (C), and fused corrected PET with CT (D). Despite breathing instructions, comparison of A and B reveals a difference in diaphragm position between CT and PET acquisition. C and D demonstrate change in shape of liver on PET after attenuation correction, to falsely match CT. Liver metastasis appears partially in lung on corrected images and results from severe loss of signal intensity in region of mismatch.

surrounding tissues in uncorrected PET images, limiting the evaluations to the diaphragmatic dome, the right lateral border, and the caudal tip of the liver. Comparison of border localizations is complicated, as liver borders appear different on uPET and acPET. For example, on acPET the level of the diaphragm shows a sharp transition from low to high uptake (lungs to liver), whereas uPET shows a transition from medium intensity in the lungs to depth-dependent variable intensity in the liver. Correction of observer-specific differences in determination of the position of the liver border on such images was based on the hypothesis that the real position of the liver border was at the mathematic middle of the measurements, which is merely an approximation. Despite correction of any systematic bias, manual localization of liver borders and focal lesions can never be perfect. Uncertainties are caused by the limited spatial and contrast resolution of PET and by interpretation difficulties on uPET images in general. Mapping of 3D ellipsoids to the liver border may reduce sampling errors to some extent but cannot fully eliminate them. Different observers may choose different points of the liver for the top, because of the sometimes irregular shape of the organ. Therefore, the interobserver variability measurements were restricted to comparison of positional differences rather than positions of liver borders, thus eliminating the variable choice of the top as a factor. Despite these considerations, the measured interobserver variabilities were all well within acceptable ranges ( $2 \mathrm{~mm}$ on average between uPET and acPET, for all borders).

Obviously, measurement of the available landmarks (3 borders in 1 direction each and a limited number of focal lesions) represents a simplification of the real situation. Only basic liver displacement will be detected, whereas local deformation and organ rotation are neglected. This leads to the conclusion that the observed misregistrations in this study may represent an underestimation rather than an overestimation and, thus, may be interpreted as an estimation of the minimal errors that occur.

The 10-mm limit for clinically relevant deviations was based on the detection limit of ${ }^{18} \mathrm{~F}$-FDG PET for small liver lesions, which has been estimated in the range of $10 \mathrm{~mm}$ (17). Thus, misregistration needs to be more than $\sim 10 \mathrm{~mm}$ to cause uncertainty in discrimination of 2 adjacent small structures. This does not imply that all cases with registration errors of $>10 \mathrm{~mm}$ will lead to misinterpretations, but awareness of the (possible) extent of misregistration may help to avoid reading errors.

The 3 studied groups were not identical, both in cohort size (i.e., $\mathrm{BH}, n=20$; FB, $n=10$; $\mathrm{SF}, n=10$ ) and in inclusion criteria (i.e., suspected liver metastases from colorectal carcinoma for $\mathrm{BH}$ and $\mathrm{SF}$ vs. various indications for $\mathrm{FB}$ ).
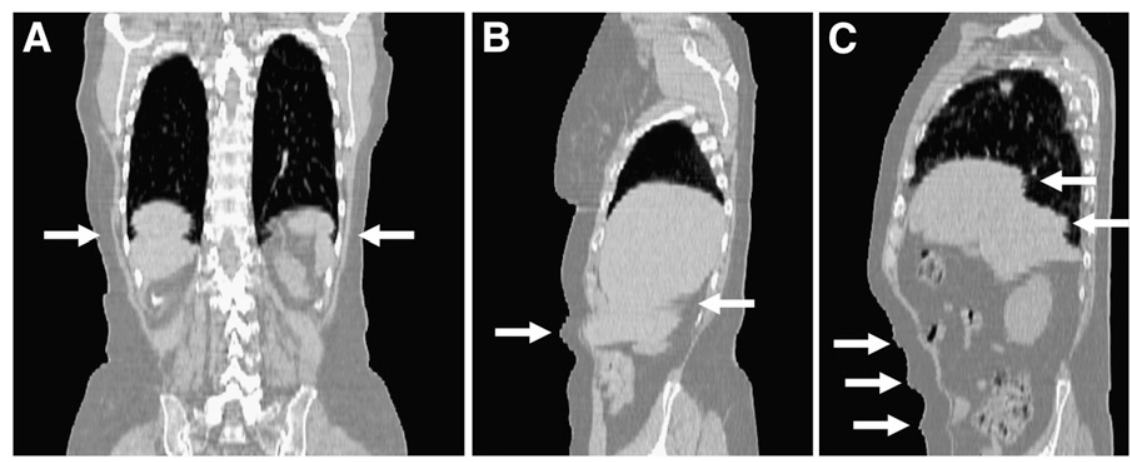

FIGURE 6. Breathing artifacts on CT: CT slices from different patients, acquired on hybrid PET/CT scanner. (A) Coronal slice of CT acquired with expiration breath-hold command. Arrows indicate artifact in middle of liver and spleen due to unsustained breath-hold. (B) Sagittal slice of CT acquired with expiration breath-hold command. Breathing commands were given relatively late and can be recognized by movement of abdominal wall (left arrow); resulting liver motion causes caudal tip of liver to appear twice (right arrow). (C) Coronal slice of CT acquired during free breathing. Breathing artifacts (arrows) are visible throughout image. 
TABLE 2

Errors and Artifacts of $>10 \mathrm{~mm}$

\begin{tabular}{|c|c|c|c|c|c|c|}
\hline \multirow[b]{3}{*}{ Errors and artifacts } & \multicolumn{4}{|c|}{ Hybrid PET/CT } & & \\
\hline & \multicolumn{2}{|l|}{ EB CT } & \multicolumn{2}{|l|}{ FB CT } & \multicolumn{2}{|l|}{ SF image } \\
\hline & Affected cases & $\%$ & Affected cases & $\%$ & Affected cases & $\%$ \\
\hline \multicolumn{7}{|l|}{ Artifacts on $\mathrm{CT}$} \\
\hline Breath-hold not sustained & $3 / 20$ & 20 & $0 / 10$ & - & $0 / 10$ & 0 \\
\hline Breath-hold timing issues & $5 / 20$ & 33 & $0 / 10$ & - & $0 / 10$ & 0 \\
\hline Free-breathing artifacts & $0 / 20$ & - & $10 / 10$ & 100 & $0 / 10$ & - \\
\hline \multicolumn{7}{|l|}{ Image registration errors $>1 \mathrm{~cm}$} \\
\hline Diaphragmatic dome & $8 / 20$ & 40 & $3 / 10$ & 30 & $0 / 10$ & 0 \\
\hline Right lateral border & $0 / 20$ & 0 & $0 / 10$ & 0 & $0 / 10$ & 0 \\
\hline Caudal tip & $11 / 20$ & 55 & $4 / 10$ & 40 & $2 / 10$ & 20 \\
\hline Individual liver lesions & $5 / 11$ & 45 & - & - & $1 / 5$ & 20 \\
\hline \multicolumn{7}{|c|}{ Attenuation-correction artifacts on PET $>1 \mathrm{~cm}$} \\
\hline Diaphragmatic dome & $10 / 20$ & 50 & $2 / 10$ & 20 & $0 / 10$ & 0 \\
\hline Right lateral border & $0 / 20$ & 0 & $0 / 10$ & 0 & $0 / 10$ & 0 \\
\hline Caudal tip & $0 / 20$ & 0 & $0 / 10$ & 0 & $0 / 10$ & 0 \\
\hline
\end{tabular}

These differences were caused by logistical issues (e.g., availability of techniques and patients), and the impact of the selection criteria on the breathing patterns of patients seems limited.

\section{Breathing Artifacts on CT}

Maintaining unforced expiration breath-hold is easily underestimated. The procedure is demanding and needs to be rehearsed before scanning. Even so, some patients will fail to sustain a breath-hold during actual scanning, causing CT artifacts (in 20\% of cases in our series). Completion of a CT scan during breath-hold cannot be guaranteed, especially when scanning elderly or diseased patients. This problem is obviously related to the acquisition time for CT during whole-body scanning, as was illustrated by the absence of artifacts on the CT images that were acquired with a fast dedicated CT scanner (for SF). Another issue is the timing of breathing instructions: $33 \%$ of cases showed deformation of the caudal part of the liver, related to the deep inspiration command when approaching the midabdomen. An earlier breath-hold command would result in an increased risk on breathing artifacts toward the end of the scan, in the upper lung fields. Increasing the speed of the $\mathrm{CT}$ acquisition would imply thicker slices for our CT scanner, thus further degrading the quality of the low-dose CT images. This issue can be avoided by performing a separate acquisition of the liver range, as exemplified by the lack of artifacts due to the breathing protocol in SF.

As expected, the alternative strategy of free superficial breathing resulted in slight $\mathrm{CT}$ artifacts throughout the liver (11). In the lungs, this effect caused small lung nodules to be missed in up to $34 \%$ of cases (18). It is unknown how this translates to imaging of the liver, but such a level of missed diagnosis will not be acceptable for correlative imaging. A faster CT scanner will result in free-breathing artifacts with a lower frequency in the images, but these artifacts cannot be eliminated entirely. Thus, with regard to CT artifacts, both breath-hold and free-breathing techniques have disadvantages, and both will benefit from a faster CT scanner.

\section{Image Registration}

During the breath-hold, the exact position of the diaphragm cannot be instructed or predicted, not even in an ideal situation (i.e., with a fast CT scanner, accurate breathhold instructions, and an exemplary patient). Furthermore, the shape of the diaphragm may differ from free breathing as during PET acquisition, because a breath-hold generates different muscle tension. This implies that differences in position and shape of the liver between PET and breathhold CT may be unavoidable. Our results confirm that registration errors of the liver are not uncommon and are rather unpredictable in extent. Misregistrations occurred primarily in the craniocaudal direction and, in most cases, were explained by insufficient expiration during the breath-hold. Deeper expiration could not be applied, because it would increase the risk of nonsustained breath-hold. Even in cases where the compliance to breath-hold instructions was perfect, registration errors of $>10 \mathrm{~mm}$ could occur, although the average extent of misregistration appeared lower. This may raise questions about our implementation of breathhold instructions. Breath-hold protocols have been evaluated previously by Goerres et al. (19). They concluded that the best breathing protocol is unforced expiration breath-hold, as performed in our study, and confirmed that the impact on image registration can still be severe (relative registration errors of -25 to $+19 \mathrm{~mm}$ vs. -16 to $+44 \mathrm{~mm}$ in our series). Brechtel et al. have reported better values for image registration at the diaphragm, but these data seem biased because evaluation was limited to acPET images only (20).

Free breathing during $\mathrm{CT}$ resulted in registration errors comparable to the $\mathrm{BH}$ protocol $(>10 \mathrm{~mm}$ in $30 \%$ and $40 \%$ 
of cases, respectively), but the maximum extent of misregistration was much lower (16 and $44 \mathrm{~mm}$, respectively). The extent of misregistration was congruent with normal liver motion during free breathing as shown by Brandner et al. (21). Nakamoto et al. (22) observed even slightly worse results at the location of the right diaphragmatic dome, with $38 \%$ misregistration of $>10 \mathrm{~mm}$ and even $10 \%$ of cases with misregistration of $>20 \mathrm{~mm}(30 \%$ and $0 \%$, respectively, in our study), with artifacts that may influence the position, shape, and apparent size of the liver on PET. Osman et al. demonstrated moderate-to-severe attenuation-correction artifacts in $18 \%$ of cases at the right diaphragmatic dome, although quantitative analysis was not performed (23). They also observed that correlation errors of liver lesions may occur, incidentally even with erroneous localization in lung instead of liver, albeit in a limited number of cases (24). Papathanassiou et al. have confirmed that lesions may be missed in liver parts that were affected by CT-based attenuation correction (25). The extent of misregistration is theoretically not dependent on the speed of the CT scanner because it "catches" the diaphragm at a random point in the breathing cycle, but this could not be evaluated in this study.

Software fusion resulted in significantly lower registration errors of both the liver, as a whole, and liver lesions. This raises the question whether such optimization of image registration and uncompromised PET image quality are possible in hybrid PET/CT. When organ-focused image registration is performed with uPET and CT images, followed by the attenuation-correction procedure, the result should be similar to SF. However, current hybrid PET/CT scanners do not provide such options. Software fusion is not ideal for high-throughput imaging, but remains useful when hybrid PET/CT is not available or for specific situations.

\section{PET Attenuation-Correction Artifacts}

Given the risk on registration errors, hybrid PET/CT with CT-based attenuation correction introduces an additional risk for artifacts on PET images. Attenuation correction will be applied erroneously on PET at the location of dense objects $(26,27)$ or where the position of a transition from low to high photon-attenuating tissue does not correspond on PET and CT $(13,22)$. The diaphragmatic area is very susceptible to such errors due to the sharp tissue/air transition, combined with the risk for positional differences. This may result in an apparent contour change of the liver on acPET images and reduced sensitivity for lesions in the affected area. On top of that, the PET signal will no longer be quantitative in the regions of attenuation-correction artifacts, which may compromise follow-up measurements.

In our series of EB imaging, deformation of the liver on acPET images was not uncommon and was rather unpredictable. The potential clinical impact is underlined by the presence of artifacts of $>10 \mathrm{~mm}$ in $50 \%$ of cases. In FB, attenuation-correction artifacts were significantly less ex- tensive but still occurred in $20 \%$ of cases with $>10 \mathrm{~mm}$. These problems must be considered unavoidable as long as registration errors occur and attenuation correction is performed with CT images. Dedicated PET does not readily have attenuation-correction artifacts, because the attenuation profile is measured using photons with energy identical to that of emission scanning, acquired during the same breathing pattern. No significant artifacts were detected in our series of dedicated PET images.

This leaves room for improvement of hybrid PET/CT image quality by reintroduction of $511-\mathrm{keV}$ transmission imaging, although this will have no impact on image registration. Development of faster and higher-quality transmission scanning - for example, simultaneous with emission scanning (28) - is eagerly awaited and is likely to prove at least as beneficial in hybrid PET/CT as the implementation of faster CT scanners.

\section{Selection of a Breathing Protocol}

Overall, free-breathing hybrid PET/CT performs somewhat better at image registration and artifacts but has poorer image correlation due to free-breathing artifacts and missing lesions on CT. Nevertheless, both approaches have been found suitable for diagnostic correlative imaging (29). When considering a breath-hold or a free-breathing hybrid PET/ $\mathrm{CT}$ protocol, it is important to realize that registration errors and attenuation-correction artifacts in breath-hold PET/CT can be recognized and circumvented afterward by adequate evaluation of UPET images, whereas missing small lesions on free-breathing CT is definitive. The final choice of technique may be guided by specific clinical questions, available equipment, individual patient characteristics, and personal preferences.

Alternative CT acquisition protocols all have disadvantages. Slow-CT, averaged cine-CT, and averaged multipleseries CT (as applied in external beam radiation therapy planning) may all severely degrade the image quality. Gated CT acquisition may provide excellent correlative imaging, at least when PET is acquired in gating identical to that of CT (30). Further experiments with such techniques need to be conducted.

Other PET radiopharmaceuticals for imaging of malignancy in the liver are likely to become available in the coming years. The presence of registration errors and attenuationcorrection artifacts in PET/CT is independent of the PET tracer, but misregistrations will be more difficult to detect for tracers with low or no uptake in normal liver tissue. This illustrates the importance of optimized imaging and reviewing, especially for imaging of novel tracers in the near future.

The combination of PET with MRI may be preferable over PET/CT - for better soft-tissue imaging characteristics and fewer radiation dose issues-but breathing issues will remain an issue. Current MRI techniques do not allow wholebody imaging during breath-hold, and free breathing during 
TABLE 3

Recommendations for Optimal PET/CT and Reviewing Recommendations to Achieve Optimal Diagnostic Quality and Interpretation of Multimodality PET/CT of Liver

\begin{tabular}{|c|c|}
\hline Category & Recommendations \\
\hline \multirow[t]{3}{*}{ Scanner requirements } & $\begin{array}{l}\text { When hybrid PET/CT with CT in expiration breath-hold is needed, a fast CT scanner (i.e., more than } \\
\text { dual slice) is preferable to avoid breath-hold compliance issues. }\end{array}$ \\
\hline & $\begin{array}{l}\text { When hybrid PET/CT with fast CT is available, expiration breath-hold CT may be preferable over } \\
\text { free-breathing CT (no missed lesions and no artifacts on CT, whereas image registration and } \\
\text { artifacts are not worse than that in free breathing). }\end{array}$ \\
\hline & $\begin{array}{l}\text { When hybrid PET/CT with slow CT is available, the choice between expiration breath-hold and } \\
\text { free-breathing CT (for nondiagnostic use) is unsettled and depends on personal preference (i.e., } \\
\text { more serious registration errors with breath-hold CT but increased risk on missed lesions on } \\
\text { free-breathing CT). }\end{array}$ \\
\hline \multirow[t]{5}{*}{ PET/CT image acquisition } & $\begin{array}{l}\text { Patient motion during or between image acquisitions may be limited by instructions and fixation } \\
\text { materials. }\end{array}$ \\
\hline & $\begin{array}{l}\text { Both expiration breath-hold and free-breathing protocols imply a trade-off between PET image } \\
\text { quality, CT image quality, and patient comfort, and selection of a technique can be based on } \\
\text { personal preference. }\end{array}$ \\
\hline & $\begin{array}{l}\text { When expiration breath-hold is to be performed, rehearsal of breathing instructions is advised } \\
\text { before actual scanning, to avoid serious misregistration and artifacts. Revert to free breathing } \\
\text { when breath-hold fails during rehearsal. }\end{array}$ \\
\hline & $\begin{array}{l}\text { Performance of PET/CT with breath-hold CT may be improved by providing feedback about image } \\
\text { registration errors to operating personnel. }\end{array}$ \\
\hline & $\begin{array}{l}\text { When reliable correlative imaging of PET and CT images without artifacts is needed on an incidental } \\
\text { basis, software fusion of dedicated PET and diagnostic CT can still be considered. }\end{array}$ \\
\hline \multirow[t]{3}{*}{ Reviewing PET/CT } & $\begin{array}{l}\text { Awareness of level of misregistration and attenuation-correction artifacts can be improved by } \\
\text { systematic reviewing of all uncorrected PET, corrected PET, and CT images. }\end{array}$ \\
\hline & $\begin{array}{l}\text { Uncorrected PET images may reveal small lesions that may be undetectable or misplaced on } \\
\text { corrected PET images, in diaphragmatic area of liver and lower lung fields. }\end{array}$ \\
\hline & $\begin{array}{l}\text { Unexplained PET lesions that show no correlating abnormalities on CT (e.g., free breathing, } \\
\text { noncontrast-enhanced or low-dose) may be resolved by correlation with separate diagnostic, } \\
\text { contrast-enhanced CT images. }\end{array}$ \\
\hline
\end{tabular}

Most recommendations will also apply to whole-body imaging with PET/CT.

MRI can severely distort the images. The best approach to hybrid PET/MRI is still unclear.

\section{CONCLUSION}

Anatomic registration errors of the liver in PET/CT may be significant, occurring primarily in the craniocaudal direction, due to breathing differences during acquisition of PET and CT, and cause subsequent attenuation-correction artifacts at the diaphragmatic dome. The extent of these errors cannot be appreciated visually on PET images that have been corrected for photon attenuation.

Breathing protocols for CT influence these issues, but no protocol can warrant perfect image registration and artifactfree images. Free-breathing PET/CT is a little less subject to these errors than breath-hold PET/CT but has unavoidable breathing artifacts throughout CT images. The choice of a breathing protocol remains a matter of personal preference. In any protocol, a faster CT scanner will reduce, but not eliminate, the chance on artifacts.

Awareness of the level of misregistration and attenuationcorrection artifacts is essential for reviewing and can be improved by consequent correlation of uncorrected PET and CT images. Furthermore, uncorrected PET images may allow detection of small lesions that became invisible or misplaced on corrected PET images, in the diaphragmatic area of the liver and the lower lung fields. On the basis of these conclusions, recommendations were formulated for optimal imaging and reviewing of integrated PET/CT (Table 3).

\section{REFERENCES}

1. Buscarini E, Savoia A, Brambilla G, et al. Radiofrequency thermal ablation of liver tumors. Eur Radiol. 2005;15:884-894.

2. Gayowski TJ, Iwatsuki S, Madariaga JR, et al. Experience in hepatic resection for metastatic colorectal cancer: analysis of clinical and pathologic risk factors. Surgery. 1994;116:703-710.

3. Blokhuis TJ, van der Schaaf MC, van den Tol MP, Comans EF, Manoliu RA, van der Sijp JR. Results of radio frequency ablation of primary and secondary liver tumors: long-term follow-up with computed tomography and positron emission tomography- ${ }^{18}$ F-deoxyfluoroglucose scanning. Scand J Gastroenterol Suppl. 2004;241:93-97.

4. Podnos YD, Henry G, Ortiz JA, et al. Laparoscopic ultrasound with radiofrequency ablation in cirrhotic patients with hepatocellular carcinoma: technique and technical considerations. Am Surg. 2001;67:1181-1184.

5. Antoch G, Vogt FM, Veit P, et al. Assessment of liver tissue after radiofrequency ablation: findings with different imaging procedures. J Nucl Med. 2005;46: $520-525$.

6. Dromain C, de Baere T, Elias D, et al. Hepatic tumors treated with percutaneous radio-frequency ablation: CT and MR imaging follow-up. Radiology. 2002;223: 255-262.

7. Kamel IR, Cohade C, Neyman E, Fishman EK, Wahl RL. Incremental value of $\mathrm{CT}$ in PET/CT of patients with colorectal carcinoma. Abdom Imaging. 2004;29: $663-668$. 
8. Selzner M, Hany TF, Wildbrett P, McCormack L, Kadry Z, Clavien PA. Does the novel PET/CT imaging modality impact on the treatment of patients with metastatic colorectal cancer of the liver? Ann Surg. 2004;240:1027-1034.

9. Veit P, Antoch G, Stergar H, Bockisch A, Forsting M, Kuehl H. Detection of residual tumor after radiofrequency ablation of liver metastasis with dualmodality PET/CT: initial results. Eur Radiol. 2006;16:80-87.

10. Vogel WV, Oyen WJG, Barentsz JO, Kaanders JHAM, Corstens FHM. PET/CT: panacea, redundancy, or something in between? J Nucl Med. 2004;45(suppl 1):15S-24S.

11. Beyer T, Antoch G, Blodgett T, Freudenberg LF, Akhurst T, Mueller S. Dual-modality PET/CT imaging: the effect of respiratory motion on combined image quality in clinical oncology. Eur J Nucl Med Mol Imaging. 2003;30: 588-596.

12. Kim JH, Czernin J, Allen-Auerbach MS, et al. Comparison between ${ }^{18} \mathrm{~F}-\mathrm{FDG}$ PET, in-line PET/CT, and software fusion for restaging of recurrent colorectal cancer. J Nucl Med. 2005;46:587-595.

13. Goerres GW, Burger C, Kamel E, et al. Respiration-induced attenuation artifact at PET/CT: technical considerations. Radiology. 2003;226:906-910.

14. Schroeder W. The Visualization Toolkit: An Object-Oriented Approach to $3 D$ Graphics. 3rd ed. New York, NY: Kitware, Inc. 2003.

15. Ibanez L, Schroeder W, Ng L, Cates J. The ITK Software Guide: The Insight Segmentation and Registration Toolkit: Version 1.4. New York, NY: Kitware, Inc. 2003.

16. van Dalen JA, Vogel W, Huisman HJ, Oyen WJ, Jager GJ, Karssemeijer N. Accuracy of rigid CT-FDG-PET image registration of the liver. Phys Med Biol. 2004;49:5393-5405.

17. Fong Y, Saldinger PF, Akhurst T, et al. Utility of ${ }^{18} \mathrm{~F}-\mathrm{FDG}$ positron emission tomography scanning on selection of patients for resection of hepatic colorectal metastases. Am J Surg. 1999;178:282-287.

18. Allen-Auerbach M, Yeom K, Park J, Phelps M, Czernin J. Standard PET/CT of the chest during shallow breathing is inadequate for comprehensive staging of lung cancer. J Nucl Med. 2006;47:298-301.

19. Goerres GW, Kamel E, Heidelberg TN, Schwitter MR, Burger C, Von Schulthess GK. PET-CT image co-registration in the thorax: influence of respiration. Eur J Nucl Med Mol Imaging. 2002;29:351-360.
20. Brechtel K, Klein M, Vogel M, et al. Optimized contrast-enhanced CT protocols for diagnostic whole-body ${ }^{18} \mathrm{~F}$-FDG PET/CT: technical aspects of single-phase versus multiphase CT imaging. J Nucl Med. 2006;47:470-476.

21. Brandner ED, Wu A, Chen H, et al. Abdominal organ motion measured using 4D CT. Int J Radiat Oncol Biol Phys. 2006;65:554-560.

22. Nakamoto Y, Tatsumi M, Cohade C, Osman M, Marshall LT, Wahl RL. Accuracy of image fusion of normal upper abdominal organs visualized with PET/CT. Eur J Nucl Med Mol Imaging. 2003;30:597-602.

23. Osman MM, Cohade C, Nakamoto Y, Wahl RL. Respiratory motion artifacts on PET emission images obtained using CT attenuation correction on PET-CT. Eur J Nucl Med Mol Imaging. 2003;30:603-606.

24. Osman MM, Cohade C, Nakamoto Y, Marshall LT, Leal JP, Wahl RL. Clinically significant inaccurate localization of lesions with PET/CT: frequency in 300 patients. J Nucl Med. 2003;44:240-243.

25. Papathanassiou D, Becker S, Amir R, Meneroux B, Liehn JC. Respiratory motion artefact in the liver dome on FDG PET/CT: comparison of attenuation correction with CT and a caesium external source. Eur J Nucl Med Mol Imaging. 2005;32:1422-1428.

26. Dizendorf E, Hany TF, Buck A, Von Schulthess GK, Burger C. Cause and magnitude of the error induced by oral CT contrast agent in CT-based attenuation correction of PET emission studies. J Nucl Med. 2003;44:732-738.

27. Goerres GW, Ziegler SI, Burger C, Berthold T, Von Schulthess GK, Buck A. Artifacts at PET and PET/CT caused by metallic hip prosthetic material. Radiology. 2003;226:577-584.

28. Matsumoto K, Kitamura K, Mizuta T, et al. Performance characteristics of a new 3-dimensional continuous-emission and spiral-transmission high-sensitivity and high-resolution PET camera evaluated with the NEMA NU 2-2001 standard. J Nucl Med. 2006;47:83-90.

29. Goerres GW, Burger C, Schwitter MR, Heidelberg TN, Seifert B, Von Schulthess GK. PET/CT of the abdomen: optimizing the patient breathing pattern. Eur Radiol. 2003;13:734-739.

30. Wolthaus JW, van Herk M, Muller SH, et al. Fusion of respiration-correlated PET and CT scans: correlated lung tumour motion in anatomical and functional scans. Phys Med Biol. 2005;50:1569-1583. 